\title{
Aging and Technology - Friends, not Foes
}

\author{
Jürgen Nehmer ${ }^{1}$, Ulman Lindenberger ${ }^{2}$, and Elisabeth Steinhagen-Thiessen ${ }^{3}$ \\ ${ }^{1}$ University of Kaiserslautern, Germany, ${ }^{2}$ Max Planck Institute for Human Development, Berlin, \\ Germany, ${ }^{3}$ Charité University Medicine Berlin, Germany
}

Technology is a friend of old age - this proposition may come as a surprise to some. That technological change in general, and modern information technology in particular, tend to place unreasonable demands on older people rather than providing support is a widespread notion. The contributions to this special issue, which stem from an interdisciplinary conference on aging and technology organized by the German Joint Academy Initiative on Aging, paint a different picture. ${ }^{1}$ They suggest that modern information technology can make significant contributions to aging more successfully.

\section{Psychological Criteria of Technological Assistance}

Sensory and cognitive abilities, such as hearing, vision, sense of balance, attention, and memory, all decline in the course of adult life and, to an increasing degree, in older years. Restrictions of physical mobility increase the risk of social isolation. Modern information technology can prevent, delay, even out, and in part mitigate the impacts of these risks and losses by training specific abilities and skills, supporting everyday behavior, and monitoring vital functions. It can contribute to elders' being in a better position to continue to lead an independent life.

In our view, modern information technology needs to fulfill three criteria to serve these functions efficiently (see Lindenberger, Lövdén, Schellenbach, Li, \& Krüger, 2008; see also Krüger, Schmidt, \& Müller, 2010):

- Criterion 1: Net resource release. The operation of technology usually requires an investment of physical and cognitive resources. The use of technology is adaptive if these operation costs are lower than the payoff associated with other changes in processing when using the technology. For example, using a mobile phone as a diary requires reading complicated instructions, so that the resource balance of this application will be low, at least initially. Objective and subjective assessments of the resource balance, however, may differ from one another: Both are relevant since perceived usefulness determines the use of the aid more strongly than its objectively demonstrable utility. At least in the medium and long term, the use of assistive technology should bring about improvements in the resource balance. Therefore, when developing and testing technical aids, we should, from the outset, closely monitor the conditions under which behavior with such aids requires fewer resources than behavior without them. This requires the integration of technological and psychological knowledge.

- Criterion 2: Person specificity. Technology can better fulfill its supportive role if it is adapted to the habits, abilities, and preferences of its individual users. Differences in performance and interests increase with aging. It is important for assistive technology to be adjusted to older users' idiosyncrasies. The earlier in life it is introduced, the easier these adjustments will be. In particular, learning to deal with assistive technology will be easier if it is introduced before the onset of physical frailty and palpable cognitive impairments. A high degree of individualization and the early application of technical aids go hand in hand.

- Criterion 3: Proximal versus distal frames of evaluation. Comprehensive assessment and the prediction of assistive technology's capacity to maintain cognitive performance and facilitate independent living are facilitated if

1 The conference, entitled “Aging, Technology, Products, Services," took place from May 29 to 31, 2008, in Berlin, Germany. It was organized by the German Joint Academy Initiative on Aging, a group of scholars, scientists, and practitioners from more than ten disciplines and fields (see Joint Academy Initiative, 2010). The Joint Academy Initiative was established by the German Academy of Sciences Leopoldina (National Academy of Sciences) in collaboration with acatech - German Academy of Science and Engineering. Its activities were funded by the Jacobs Foundation Zurich. 
the entire lifespan is considered and historical change is taken into account. When they eventually reach the age of 80, today's 30-year-olds will be using multifunctional mobile devices much differently than today's 80-yearolds use currently available mobile phones. Moreover, a critical assessment of the use and risks of technical aids may yield different results depending on whether their effects are observed over a short or a longer period. For instance, the use of mobile navigation systems in automobiles can help people to reach their destination more efficiently, that is, faster and with less mental effort, and they allow them to make use of their freed-up cognitive resources to have a conversation or follow an audiobook while driving. But it is at least conceivable, albeit (yet) unproven, that the constant use of a navigation system while driving leads to chronic disuse of navigational and spatial orientation skills. The Seattle Longitudinal Study, for example, showed that cohorts born later were not as good at mental arithmetic as those born earlier, despite the fact that, generally speaking, the later cohorts' cognitive functioning tended to be better than that of their predecessors' (Schaie, 1996). The decline in mental arithmetic was probably connected to the introduction of pocket calculators in school.

\section{The Interplay Between Self-Initiated Processing and Environmental Support}

The employment of technology may improve the use of existing resources and influence their lifespan development. As is often true when attempting to enhance behavioral performance and development, the important task is to strike the right balance between environmental support and self-initiated processing (Craik, 1983). Modern information technology can tune the degree of support provided to individual users, and lower or raise it according to their needs. In this manner, the degree of difficulty in coping with everyday life can be kept in a balance between cognitive underload and overload that favorably influences further cognitive development in old age.

At present, the impression often arises that older people have to adapt to the requirements of technology, consolidating the widely held belief that old age and modern technology are antagonistic. However, just the opposite makes sense - and is also technically feasible. Older people are "experts on their own lives" and have a wealth of knowledge about their personal preferences, habits, and idiosyncrasies. Sometimes, however, it is difficult for them to apply this knowledge adequately, for example, when they are tired, distracted, have to pursue several goals at once, or when their senses and bodily functions consume their cognitive resources. In such situations, flexibly assistive technologies ought to provide reliable cues that support people in keeping track of their goals and performing intended actions appropriately.

\section{Overview of the Special Issue}

The contributions to this special issue interrogate the relationship between aging and technology from a variety of different perspectives. Accordingly, this issue is structured into four sections. In the first, Technology in Healthy Aging, Schmiedek, Bauer, Lövdén, Brose, and Lindenberger (2010) describe a computer-based cognitive training program that has been successfully implemented in a largescale, age-comparative study on intraindividual variability and cognitive plasticity. Objective measures and retrospective self-report evaluations demonstrate the program's feasibility and acceptance. Schellenbach, Lövdén, Verrel, Krüger, and Lindenberger (2010) examine how different assistive navigation technologies affect walking regularity and navigation performance in younger and older adults. The authors conclude that assistive navigation devices show promise in supporting older adults' pedestrian mobility if aging-induced increments in cognitive demands of spatial navigation and postural control are considered.

In the following section, Prevention, Rehabilitation, and Coping with Disease, Gövercin, Missala, Marschollek, and Steinhagen-Thiessen (2010) provide an overview of clinical trials investigating the effectiveness of virtual rehabilitation (VR) and telerehabilitation (TR) for upper-limb recovery after stroke, and conclude that both approaches are promising. Nehmer, Becker, Kleinberger, and Prückner (2010) describe electronic systems designed to assist people in their everyday life, focusing on ambient intelligence technology. Based on an emergency safeguard system developed in their lab, they conclude that ambient intelligence technology has the potential to promote independent living.

In a futuristic section, Technological Trends: Aging in 20 Years, Krüger et al. (2010) provide a detailed account of smart environments and artifacts that are able to perceive the users' context and activity, anticipate their needs, and act to provide proactive support. They convincingly argue that ubiquitous computing technologies of this kind possess the potential to increase autonomy and independence. Chang et al. (2010) describe a pioneering technology, silicon dry electrodes based on Micro-Electro-Mechanical Systems, and its use for detecting drowsiness and fatigue in real-life settings, such as driving. The system may be particularly helpful for older drivers who are affected by periods of tiredness and fatigue.

In the concluding section, Economic and Societal Implications, de Ruyter, Zwartkruis-Pelgrim, and Aarts (2010) report on three projects from the research environment CareLab and discuss some of the challenges crucial to the success of assistive technology. Finally, Krieg-Brückner, Röfer, Shi, and Gersdorf (2010) summarize how intelligent mobility assistants developed in the Bremen Ambient As- 
sisted Living Lab on mobility, such as the Rolland wheelchair and the iWalker walker, compensate for the decreasing physical and cognitive capabilities of their users.

Altogether, the contributions to this special section make a strong case for the beneficial effects of modern technology on aging individuals and societies.

\section{Concluding Remarks}

Technical innovations for successful aging rely on research collaboration between computer scientists, psychologists, engineers, interior designers, architects, area planners, and medical scientists. They offer opportunities both for older people and for societies with growing numbers of old and very old people. Technology applied in a flexible and supportive manner can improve the balance between support and challenge in old age, enhance everyday skills, and strengthen participation in social life, with positive effects on performance, well-being, and self-esteem. It can also reduce costs in the health and social security system and create a potential for economic growth by improving and extending the ability to lead an independent life. And last but not least, the inventiveness of the users of technical innovations who are themselves aging will help to ensure that the opportunities outweigh the risks.

\section{Acknowledgments}

We would like to thank all contributors to this special issue. We thank James Lattimer for improving the English and express our special gratitude to Dr. Julia Delius and Dipl.Inf. Michael Schellenbach for their excellent editorial assistance.

\section{References}

Chang, C.-W., Ko, L.-W., Lin, F.-C., Su, T.-P., Jung, T.-P., Lin, C.-T., \& Chiou, J.-C. (2010). Drowsiness monitoring with EEG-based MEMS biosensing technologies. GeroPsych, 23, 105-111. doi: 10.1024/1662-9647/a000014.

Craik, F. I. M. (1983). On the transfer of information from temporary to permanent memory. Philosophical Transactions of the Royal Society of London, B302, 341-359. doi: 10.1098/rstb.1983.0059

de Ruyter, B., Zwartkruis-Pelgrim, E., \& Aarts, E. (2010). Ambient assisted living research in the CareLab. GeroPsych, 23, 113-117. doi: 10.1024/1662-9647/a000015.

Gövercin, M., Missala, I. M., Marschollek, M., \& Steinhagen-Thiessen, E. (2010). Virtual rehabilitation and telerehabilitation for the upper limb: A geriatric review. GeroPsych, 23, 77-88. doi: 10.1024/1662-9647/a000011.

Joint Academy Initiative on Aging. (2010). More years, more life:
Recommendations of the Joint Academy Initiative on Aging. Nova Acta Leopoldina, 108(372).

Krieg-Brückner, B., Röfer, T., Shi, H., \& Gersdorf, B. (2010). Mobility assistance in the Bremen Ambient Assisted Living Lab. GeroPsych, 23, 119-128. doi: 10.1024/1662-9647/a000009.

Krüger, A., Schmidt, A., \& Müller, J. (2010). Technological and research perspectives of old-age ubiquitous computing. GeroPsych, 23, 97-103. doi: 10.1024/1662-9647/a000012.

Lindenberger, U., Lövdén, M., Schellenbach, M., Li, S.-C., \& Krüger, A. (2008). Psychological principles of successful aging technologies: A mini-review. Gerontology, 54, 59-68. doi: $10.1159 / 000116114$.

Nehmer, J., Becker, M., Kleinberger, T., \& Prückner, S. (2010). Electronic emergency safeguards: Sensor-based detection and prevention of critical health conditions. GeroPsych, 23, 89-96. doi: 10.1024/1662-9647/a000008.

Schaie, K. W. (1996). Adult intellectual development: The Seattle Longitudinal Study. New York: Cambridge University Press.

Schellenbach, M., Lövdén, M., Verrel, J., Krüger, A., \& Lindenberger, U. (2010). Sensorimotor-cognitive couplings in the context of assistive spatial navigation for older adults. GeroPsych, 23, 67-75. doi: 10.1024/1662-9647/a000010.

Schmiedek, F., Bauer, C., Lövdén, M., Brose, A., \& Lindenberger, U. (2010). Cognitive enrichment in old age: Web-based training programs. GeroPsych, 23, 59-65. doi: 10.1024/16629647/a000013.

Jürgen Nehmer

University of Kaiserslautern

FB Informatik

Gottlieb-Daimler-Str.

P. O. Box 3049

D-67663 Kaiserslautern

Germany

E-mail: nehmer@informatik.uni-kl.de

Ulman Lindenberger

Max Planck Institute for Human Development

Lentzeallee 94

D-14195 Berlin

E-mail: seklindenberger@mpib-berlin.mpg.de

Elisabeth Steinhagen-Thiessen

Geriatrics Research Group

Department of Geriatric Medicine

Charité University Medicine Berlin

Reinickendorfer Straße 61

D-13347 Berlin

Germany

E-mail: elisabeth.steinhagen-thiessen@ charite.de 